\title{
VOLUME BOUNDS FOR GENERALIZED TWISTED TORUS LINKS
}

\author{
Abhijit Champanerkar, David Futer, Ilya Kofman, Walter Neumann \\ AND JessicA S. PURCELL
}

\begin{abstract}
Twisted torus knots and links are given by twisting adjacent strands of a torus link. They are geometrically simple and contain many examples of the smallest volume hyperbolic knots. Many are also Lorenz links.

We study the geometry of twisted torus links and related generalizations. We determine upper bounds on their hyperbolic volumes that depend only on the number of strands being twisted. We exhibit a family of twisted torus knots for which this upper bound is sharp, and another family with volumes approaching infinity. Consequently, we show there exist twisted torus knots with arbitrarily large braid index and yet bounded volume.
\end{abstract}

\section{Introduction}

Recently, there has been interest in relating the volume of a hyperbolic knot and link to other link properties. Lackenby has related the volume of an alternating link to the number of twist regions in its diagram [13], and this relationship was extended to larger classes of links that satisfy a certain threshold of complexity, such as a high amount of symmetry or twisting $[10,11,14]$. To better understand volumes in general, it seems natural to also investigate properties of knots and links that are "simple."

Twisted torus knots and links are obtained by twisting a subset of strands of a closed torus braid. These knots are geometrically simple by several different measures of geometric complexity. Dean [9] showed that they often admit small Seifert fibered and lens space fillings. In [6,7], it was discovered that twisted torus knots dominate the census of "simplest hyperbolic knots," those whose complements can be triangulated with seven or fewer ideal tetrahedra. It is not surprising then that twisted torus knots contain many examples of the smallest volume hyperbolic knots.

In this paper, we investigate the geometry of twisted torus links and closely related generalizations. We determine upper bounds on their volumes in terms of their description parameters. We also exhibit a family of twisted torus knots for which this upper bound is sharp, and another family with volumes approaching infinity.

A consequence of these results is that the braid index of a knot or link gives no indication of its volume. Using techniques of [4] to determine braid index, we show there exist twisted torus knots with arbitrarily large braid index and yet bounded volume. The reverse result is also known, for example, closed 3-braids can have unbounded volume [12].

Received by the editors 18 July, 2010. 


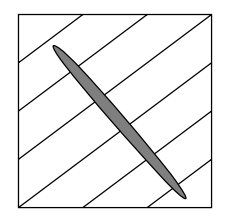

Figure 1. The regular neighborhood of a line segment crossing $r$ strands.

1.1. Twisted torus links. A positive root $\beta$ will mean a positive $n$-braid whose $n$th power is the central element $\Delta_{n}^{2}$ (i.e., the full twist) in $B_{n}$. In Theorem 2.1, we show that there are $2^{n-2}$ braid isotopy classes of positive $n$th roots, all of which have the form

$$
\beta=\sigma_{i_{1}} \cdots \sigma_{i_{n-1}}
$$

with $i_{1}, \ldots, i_{n-1}$ a permutation of $1, \ldots, n-1$, and all of which are conjugate in $B_{n}$ to $\delta_{n}=\sigma_{1} \cdots \sigma_{n-1}$, which we will call the standard root. Let $\bar{\delta}_{n}=\sigma_{n-1} \cdots \sigma_{1}$ denote a conjugate root which we will also use below.

Definition 1.1. Let $p>0, q, s \neq 0$, and $1<r \leq p+|q|$ be integers. Let $\beta \in B_{r}$ be any positive root. Let $L$ be a $(p, q)$-torus link embedded on a flat torus. Let $D$ be a regular neighborhood of a line segment that crosses $r$ strands of $L$, as in Figure 1. The twisted torus link $T(p, q, r, s, \beta)$ is formed from $L$ by replacing the $r$ strands of $L \cap D$ with the braid $\beta^{s}$. We will usually suppress the $\operatorname{root} \beta$ in the notation.

In [9], Dean defined the twisted torus link $T(p, q, r, s)$ by replacing the $r$ strands of $L \cap D$ with the $r$-braid $\left(\delta_{r}\right)^{s}$, for $s$ an integer multiple of $r$, which implies $T(p, q, r, s)=$ $T(q, p, r, s)$. In [6, 7], twisted torus links were defined as closed braids for $1<r<p$ and for $s$ an integer multiple of $r$; namely, the closure of the $p$-braid $\left(\delta_{p}\right)^{q}\left(\delta_{r}\right)^{s}=$ $\left(\sigma_{1} \cdots \sigma_{p-1}\right)^{q}\left(\sigma_{1} \cdots \sigma_{r-1}\right)^{s}$. In [4], this was generalized to any integer $s$, for which switching $p$ and $q$ may result in distinct links. Definition 1.1 includes all of these as special cases.

1.2. $\boldsymbol{T}$-links. A natural way to generalize twisted torus links is to repeatedly twist nested subsets of strands.

Definition 1.2. Let $r_{1}>\cdots>r_{k} \geq 2$ and $q, s_{i} \neq 0$ be integers. Define the $T$-link $T\left((p, q),\left(r_{1}, s_{1}, \beta_{1}\right), \ldots,\left(r_{k}, s_{k}, \beta_{k}\right)\right)$ to be formed from $L$, as in Definition 1.1 with $r=r_{1}$, by replacing the $r$ strands of $L \cap D$ with the braid $\beta_{1}^{s_{1}} \cdots \beta_{k}^{s_{k}}$, where each $\beta_{i}$ is a specified $r_{i}$-th root of the full twist on the first $r_{i}$ strands. We will usually suppress the roots $\beta_{i}$ in the notation. If $k=1$, a $T$-link is a twisted torus link. See Figure 2 for an example.

The above definition of $T$-links is generalized from the definition in [4], but our $s_{i}$ 's may be negative and our $r_{i}$ 's form a decreasing sequence. By [4], it follows that positive $T$-links using only the roots $\bar{\delta}_{r_{i}}$ coincide with Lorenz links. The $T$-link point of view opens the door to understanding the geometry of Lorenz link complements. But our results below do not require positivity. We provide volume bounds for $T$-links, so in particular, for Lorenz links. 


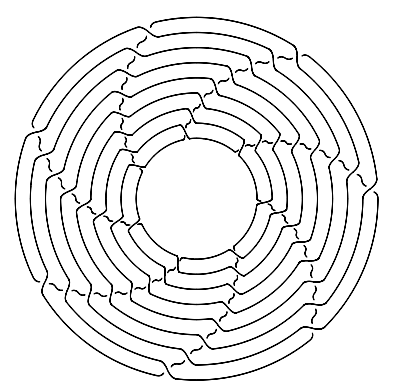

(a) $T(9,7)$

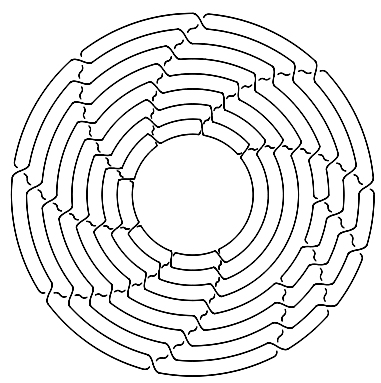

(b) $T(9,7,5,3)$

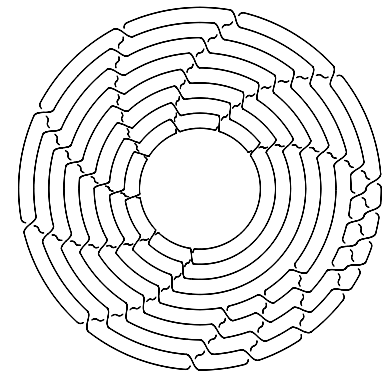

(c) $T((9,7),(5,3),(3,4))$

Figure 2. A torus knot, a twisted torus knot, and a $T$-knot using standard roots.

1.3. Volume bounds. Let $\operatorname{Vol}(K)$ denote the volume of the link complement $S^{3} \backslash K$. If $K$ is not hyperbolic, $\operatorname{Vol}(K)$ is the sum of volumes of the hyperbolic pieces of $S^{3} \backslash K$. Let $v_{3} \approx 1.0149$ denote the volume of the regular hyperbolic ideal tetrahedron. In Section 3, we prove the following result.

Theorem 1.3. Let $T(p, q, r, s)$ be a twisted torus link with positive root $\beta$. Then

$$
\begin{aligned}
& \operatorname{Vol}(T(p, q, r, s))<10 v_{3}, \quad \text { if } r=2, \\
& \operatorname{Vol}(T(p, q, r, s))<v_{3}(2 r+10), \quad \text { if } s \bmod r=0, \\
& \operatorname{Vol}(T(p, q, r, s))<v_{3}\left(r^{2}+r+10\right), \quad \text { if } \beta=\delta_{r} \text { or } \bar{\delta}_{r}, \\
& \operatorname{Vol}(T(p, q, r, s))<v_{3}\left(r^{2}+4 r+4\right), \quad \text { otherwise. }
\end{aligned}
$$

Theorem 1.4. Choose any sequence $\left(p_{N}, q_{N}\right) \rightarrow(\infty, \infty)$, such that $\operatorname{gcd}\left(p_{N}, q_{N}\right)=1$. Then the twisted torus knots $T\left(p_{N}, q_{N}, 2,2 N\right)$ have volume approaching $10 v_{3}$ as $N \rightarrow \infty$.

The noteworthy feature of Theorem 1.3 is that the upper bound only depends on the parameter $r$. The independence of $p$ and $q$ was a surprise to the authors. One consequence of this independence is that there is no direct relationship between the braid index and volume of a link.

Corollary 1.5. The twisted torus knots $T(p, q, 2, s), p, q>2, s>0$, have arbitrarily large braid index and volume bounded by $10 v_{3}$.

Proof. For $q, s>0$ and $p>r$, the minimal braid index of $T(p, q, r, s)$ with root $\delta_{r}$ or $\bar{\delta}_{r}$ is exactly $\min (p, q)$ if $r \leq q$, and $\min (s+q, r)$ if $r \geq q$ (Corollary 8 of [4]). By Theorem 1.3, any $T(p, q, 2, s)$ has volume bounded above by $10 v_{3}$, but for all $p, q>2$, its minimal braid index is $\min (p, q)$.

The reverse of Corollary 1.5 is also true: for example, closed 3-braids have unbounded hyperbolic volume. See [12, Theorem 5.5].

When the twisted torus knots are Lorenz, we can use Lorenz duality [4, Corollary 4] to obtain another volume bound in terms of $q$. 
Corollary 1.6. If we use the roots $\delta_{r}$ or $\bar{\delta}_{r}$, and let $(q \cdot s)>0$ and $p>r$,

$$
\begin{aligned}
& \operatorname{Vol}(T(p, q, r, s))<10 v_{3}, \quad \text { if } q=2, \\
& \operatorname{Vol}(T(p, q, r, s))<v_{3}(2|q|+10), \quad \text { if } p \bmod q=r, \\
& \operatorname{Vol}(T(p, q, r, s))<v_{3}\left(q^{2}+|q|+10\right), \quad \text { otherwise. }
\end{aligned}
$$

Proof. The braids $\left(\delta_{p}^{q} \delta_{r}^{s}\right),\left(\bar{\delta}_{p}^{q} \bar{\delta}_{r}^{s}\right),\left(\delta_{r}^{s} \delta_{p}^{q}\right)$ and $\left(\bar{\delta}_{r}^{s} \bar{\delta}_{p}^{q}\right)$ have isotopic closures. When $(q \cdot s)>0$, all the twisting is in the same direction. Thus, under the hypotheses of the corollary, the twisted torus links are Lorenz or mirror images of Lorenz links. So they satisfy the following duality coming from the symmetry of the Lorenz template $[4]$ :

$$
T(p, q, r, s)=T(q+s, r, q, p-r) \text { if } q, s>0 .
$$

In particular, $q$ and $r$ can be exchanged.

Our results extend to volume bounds for $T$-links.

Theorem 1.7. If $L$ is the $T$-link $T\left((p, q),\left(r_{1}, s_{1}, \beta_{1}\right), \ldots,\left(r_{k}, s_{k}, \beta_{k}\right)\right)$,

$$
\begin{aligned}
& \operatorname{Vol}(L)<v_{3}\left(r_{1}^{2}+9 r_{1}-8\right), \quad \text { if all } s_{i} \bmod r_{i}=0 \\
& \operatorname{Vol}(L)<v_{3}\left(\frac{1}{3} r_{1}^{3}+\frac{5}{2} r_{1}^{2}+5 r_{1}-5\right), \quad \text { otherwise. }
\end{aligned}
$$

Again, the notable feature of Theorem 1.7 is that even though it takes many parameters to specify a $T$-link, a single coordinate suffices to bound the volume from above.

If $p>r_{1}$, the braids $\left(\bar{\delta}_{p}^{q} \bar{\delta}_{r_{1}}^{s_{1}} \cdots \bar{\delta}_{r_{k}}^{s_{k}}\right)$ and $\left(\delta_{r_{k}}^{s_{k}} \cdots \delta_{r_{1}}^{s_{1}} \delta_{p}^{q}\right)$ have isotopic closures. So if all $s_{i}>0$ and all roots are $\bar{\delta}_{r_{i}}$, these $T$-links are Lorenz by [4], and Lorenz duality implies a result analogous to Corollary 1.6, with $r_{1}$ replaced by $\left(q+s_{1}+s_{2}+\cdots+s_{k-1}\right)$.

If $r_{1} \leq d=\operatorname{gcd}(p, q)$ then $T\left((p, q),\left(r_{1}, s_{1}\right), \ldots,\left(r_{k}, s_{k}\right)\right)$ is a satellite link with companion $T(p / d, q / d)$. In the JSJ decomposition of this link complement, only the solid torus minus $T\left(\left(r_{1}, s_{1}\right), \ldots,\left(r_{k}, s_{k}\right)\right)$ can have non-zero volume, which is bounded by the function from Theorem 1.7 with $r_{1}$ replaced by $r_{2}$. Similarly, if $r \leq \operatorname{gcd}(p, q)$ in Theorem 1.3 the volume is zero. So we assume from now on that $r_{1}>\operatorname{gcd}(p, q)$, resp. $r>\operatorname{gcd}(p, q)$.

In Section 4, we prove the following theorem, which shows that these volume bounds are non-trivial.

Theorem 1.8. For any number $V$, there exists a hyperbolic twisted torus knot whose complement has volume at least $V$.

In the proof of Theorem 1.8, we construct links by twisting along annuli. This theorem can be compared with related work of Baker [3]. He showed that the class of Berge knots, which contains some twisted torus knots, contains knots which have arbitrarily large volume. However, Baker's examples are not necessarily twisted torus knots. Nor are the examples produced to prove Theorem 1.8 necessarily Berge knots. 


\section{Positive roots of the full twist in $B_{n}$}

Twisting is a natural geometric operation on links because any full twist comes from \pm 1 Dehn surgery on an unknot in $S^{3}$. However, to define a twisted torus link without full twists, as in Definition 1.1, we must first choose a particular root of the full twist. The link type generally changes with different choices of roots. Braids provide a natural notation for this choice, and in this section we give an elementary proof of the classification of braid isotopy classes of roots in Theorem 2.1. This result also follows from Corollary 12 of [5], which uses the Garside structure of the braid group.

Recall that a positive root $\beta$ is a positive $n$-braid whose $n$th power is $\Delta^{2}$ in $B_{n}$. Since $\Delta^{2}$ has length $n(n-1)$ in the braid generators, a positive root must have length $n-1$. Moreover, since all $n-1$ generators must be involved, $\beta$ must have the form

$$
\beta=\sigma_{i_{1}} \ldots \sigma_{i_{n-1}}
$$

with $i_{1}, \ldots, i_{n-1}$ a permutation of $1, \ldots, n-1$. This list of $(n-1)$ ! braid words includes all positive roots, but with multiplicity because many of these are isotopic braids.

Theorem 2.1. There are $2^{n-2}$ braid isotopy classes of positive nth roots, all of which are conjugate in $B_{n}$ to the standard root $\delta_{n}=\sigma_{1} \cdots \sigma_{n-1}$.

Proof. Using the fact that $\sigma_{i} \sigma_{j}=\sigma_{j} \sigma_{i}$ whenever $|i-j| \geq 2$, we can move any $\sigma_{i_{k}}$ with $i_{k}<i_{k-1}$ to the left in the above expression unless $i_{k}=i_{k-1}-1$. After repeating this until no further such moves are possible, the indices $i_{1}, i_{2}, \ldots, i_{n-1}$ will form the concatenation of some number $(r+1)$ of monotone decreasing chains,

$$
j_{1}, j_{1}-1, \ldots, 1 ; j_{2}, j_{2}-1, \ldots, j_{1}+1 ; j_{3}, j_{3}-1, \ldots, j_{2}+1 ; \ldots ; n-1, n-2, \ldots, j_{r}+1
$$

This gives a normal form for $\beta$ up to braid isotopy that is determined by $\left\{j_{1}, j_{2}, \ldots, j_{r}\right\}$ which is a subset of $\{1, \ldots, n-2\}$. Every subset corresponds to a normal form expression, so there are $2^{n-2}$ possible normal form expressions. (The empty set corresponds to the chain $n-1, n-2, \ldots, 1$.)

We claim that these normal forms give $2^{n-2}$ different roots. Indeed, a simple calculation shows that their images in the permutation group $S_{n}$ are distinct, so they are distinct.

Now, identify braids in this list if they are equivalent under cyclic permutation plus the braid relation $\sigma_{i} \sigma_{j}=\sigma_{j} \sigma_{i}$ for $|i-j| \geq 2$. It is easy to see that every braid given in the normal form is cyclically equivalent to either $\delta_{n}$ or $\bar{\delta}_{n}=\sigma_{n-1} \cdots \sigma_{1}$. Moreover, $\bar{\delta}_{n}$ is conjugate to $\delta_{n}$ by $\Delta$. Therefore, every braid given by this normal form is conjugate to $\delta_{n}$, and hence is an $n$th root.

From a picture, it becomes clear that these braids are roots. We illustrate this in Table 1. In this table, braids should be read counterclockwise, starting at $120^{\circ}$ on the circle.

The following lemma is immediate from the normal form for $\beta$, particularly the form of the indices $i_{1}, i_{2}, \ldots, i_{n-1}$ in equation (2.1). We record it here, since we will use it in the next section. 
TABLE 1. All eight fifth roots, repeated three times in each diagram. The corresponding braidword and subset $\left\{j_{1}, \ldots, j_{r}\right\}$ are given below each one

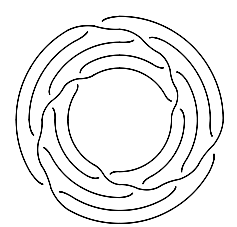

4321

$\{\emptyset\}$

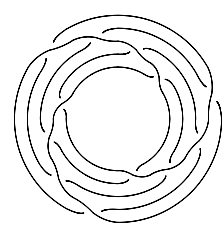

1432

$\{1\}$

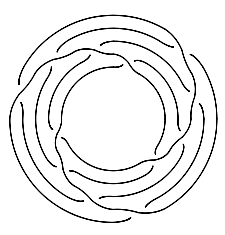

2143

$\{2\}$

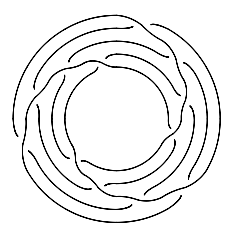

3214

$\{3\}$

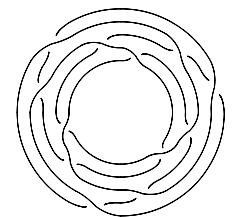

1243

$\{1,2\}$

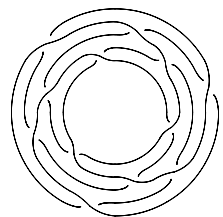

1324

$\{1,3\}$

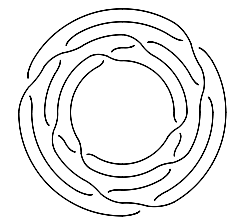

2134

$\{2,3\}$

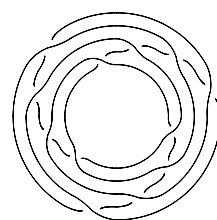

1234

$\{1,2,3\}$

Lemma 2.2. In the normal form for $\beta=\sigma_{i_{1}} \sigma_{i_{2}} \cdots \sigma_{i_{n-1}}$ :

(a) The generator $\sigma_{j}$ appears before $\sigma_{j-1}$ and $\sigma_{j+1}, 1<j<n-1$, if and only if the index $j$ is the first entry of a decreasing chain of length at least two in equation (2.1).

(b) Similarly, $\sigma_{j}$ appears after both $\sigma_{j-1}$ and $\sigma_{j+1}$ if and only if the index $j$ is the last entry of a decreasing chain of length at least two in equation (2.1).

(c) The generator $\sigma_{1}$ appears before $\sigma_{2}$ if and only if $\sigma_{1}$ is the first generator of the braid word, with the index 1 forming a chain of length one. The generator $\sigma_{1}$ appears after $\sigma_{2}$ if and only if the index 1 is the last entry of a decreasing chain of length at least two in equation (2.1).

(d) The generator $\sigma_{n-1}$ appears before $\sigma_{n-2}$ if and only if the index $(n-1)$ is first in a chain of length at least two; and $\sigma_{n-1}$ appears after $\sigma_{n-2}$ if and only if $\sigma_{n-1}$ is the last generator in the braid word, with the index $(n-1)$ forming a chain of length one.

\section{Upper volume bounds}

In this section, we prove Theorems 1.3, 1.4 and 1.7, establishing upper bounds on the volumes of twisted torus links and $T$-links.

3.1. Twisted torus links. First, we define $M(p, q, r, s)$, which is a surgery parent manifold to $T(p, q, r, s)$.

Definition 3.1. Start with integers $p, q>0$ and $r$ so that $0<r \leq p+q$. Let $C_{1}$ be an unknot in $S^{3}$, so $S^{3} \backslash C_{1}$ is the solid torus $S^{1} \times D^{2}$, where we view $D^{2}$ as the unit disk. Let $C_{2}$ be the core of this solid torus, $C_{2}=S^{1} \times\{0\}$. Let $T(p, q)$ be the 
$(p, q)$-torus link sitting on $S^{1} \times S_{1 / 2}^{1}$, where $S_{1 / 2}^{1}$ is the circle at radius $1 / 2$ in the disk $D^{2}$. Augment $T(p, q)$ with an unknotted circle $L$ that encircles the first $r$ strands. For any given positive root $\beta \in B_{r}$, and integer $s$, replace the $r$ strands of $T(p, q)$ encircled by $L$ with the braid $\beta^{s}$, and call the result $K(p, q, r, s, \beta)$. Let $M(p, q, r, s)$ be the link complement $S^{3} \backslash\left(C_{1} \cup C_{2} \cup L \cup K(p, q, r, s, \beta)\right)$. (As usual, we suppress $\beta$.)

Observe the following facts about $M(p, q, r, s)$. Let $I=(-1,1)$. First, since $C_{1} \cup C_{2}$ is the Hopf link, $S^{3} \backslash\left(C_{1} \cup C_{2}\right) \cong T^{2} \times I$. Hence, $M(p, q, r, s)$ is homeomorphic to the complement of a link of at least two components in $T^{2} \times I$, one component corresponding to $L$, and the others to $K(p, q, r, s, \beta)$ of Definition 3.1. We will illustrate examples of $M(p, q, r, s)$ by drawing links in $T^{2} \times I$. We will also use the framings induced from $T^{2} \times\{0\}$ on $C_{1}$ and $C_{2}$.

Second, the twisted torus link $T(p, q, r, s)$ is obtained from $M(p, q, r, s)$ by Dehn filling along slopes $(0,1)$ and $(1,0)$ on $C_{1}$ and $C_{2}$, respectively, and along the slope $(1,0)$ on $L$. More generally, we obtain a twisted torus link of the form $T\left(p^{\prime}, q^{\prime}, r\right.$, $N r+s)$ by Dehn filling $L$ along the slope $(1, N)$ for any integer $N$, and Dehn filling $C_{1}$ and $C_{2}$ along slopes with geometric intersection number 1 . For example, see [15]. Since volume only decreases under Dehn filling [16], we wish to bound the volume of $M(p, q, r, s)$.

Finally, the homeomorphism type of $M(p, q, r, s)$ is summarized by the following lemma.

Lemma 3.2. If $r>\operatorname{gcd}(p, q), M(p, q, r, s)$ is homeomorphic to $M\left(n, m, r, s^{\prime}\right)$ or $M\left(m, n, r, s^{\prime}\right)$ where $s^{\prime}=s \bmod r, 0 \leq s^{\prime}<r$, and $n$ and $m$ come from a truncated continued fraction expansion of $p / q$. Precisely, $n$ and $m$ are integers satisfying $0<$ $n<r, 0<m<r, n+m \geq r$, and

$$
\frac{p}{q}=a_{0}+\frac{1}{a_{1}+\frac{1}{\ddots \frac{1}{a_{k}+m / n}}} .
$$

where $a_{i}$ are positive integers for $1 \leq i \leq k$.

Proof. First, since $L$ is an unlink encircling the $r$ strands of the braid $\beta^{s}, M(p, q, r, s)$ is homeomorphic to $M(p, q, r, s+j r)$ for any integer $j$. Thus in particular it is homeomorphic to $M\left(p, q, r, s^{\prime}\right)$ where $s^{\prime}=s \bmod r$ and $0 \leq s^{\prime}<r$.

Next, since $M(p, q, r, s)$ is a link complement in $T^{2} \times I$, it will be homeomorphic to the link complement obtained by Dehn twisting an integer number of times about the meridian or longitude of $T^{2}$. Thus applying the (truncated) Euclidean algorithm to the slope $p / q$ on $T^{2}$, we may reduce the slope to some $m / n$ with $m<r, n<r$, $m+n \geq r$. The process gives the truncated continued fraction expansion of $p / q$.

Figure 3 illustrates the proof of Lemma 3.2 when $p=3, q=7, r=5$, and $s=0$. In that figure, Dehn twist about the horizontal curve $(0,1)$, and isotope to obtain $M(3,4,5,0)$.

Our goal is to bound the simplicial volume of $M(p, q, r, s)$. Recall that for any compact 3-manifold $M$, whether closed or with boundary, the simplicial volume is defined to be $\operatorname{Vol}(M)=v_{3}\|M\|$, where $\|M\|$ is the Gromov norm of $M$. See $[16$, 

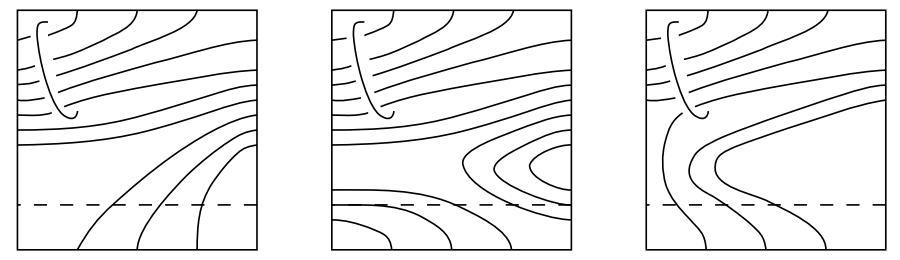

Figure 3. $M(3,7,5,0)$ is homeomorphic to $M(3,4,5,0)$.

Chapter 6] for background on the Gromov norm. For our purposes, we will need three properties:

(1) $\|M\|$ is bounded above by the number of (compact or ideal) tetrahedra needed to triangulate $M$.

(2) $\|M\|$ is non-increasing under Dehn filling.

(3) If $M$ is hyperbolic, then $v_{3}\|M\|$ is the hyperbolic volume of $M$. Thus there is no ambiguity in the notation $\operatorname{Vol}(M)$.

Combining these properties, we conclude that $v_{3}$ times the number of ideal tetrahedra in a triangulation of $M(p, q, r, s)$ provides an upper bound on the volume of any of its hyperbolic Dehn fillings. This upper bound applies regardless of whether $M(p, q, r, s)$ is hyperbolic.

The following straightforward lemma will assist us in counting the number of tetrahedra in an ideal triangulation of $M(p, q, r, s)$.

Lemma 3.3. Two pyramids glued along a common base, a polygon with $t$ sides, may be subdivided into t tetrahedra. In the case $t=3$, the pyramids may be subdivided into 2 tetrahedra.

Proof. If $t=3$, each pyramid is a tetrahedron, and there is nothing to prove. If $t>3$, then remove the base polygon, and add an edge running between the two tips of the pyramids. Perform stellar subdivision, obtaining one tetrahedron for each of the $t$ edges of the base polygon.

Lemma 3.4. Let $n, m$, and $r$ be integers such that $n<r, m<r$, and $n+m \geq r$. The manifold $M(n, m, r, 0)$ can be decomposed into $t$ ideal tetrahedra, where

$$
t= \begin{cases}10, & \text { if } r=2, \\ 2 r+8, & \text { if } n+m=r \text { and } r>2 \\ 2 r+10, & \text { otherwise. }\end{cases}
$$

Proof. Recall $M(n, m, r, 0)$ is the complement of a link in $T^{2} \times(-1,1)$, with the torus link $T(n, m)$ on $T^{2} \times\{0\}$. Put $L$ perpendicular to $T^{2} \times\{0\}$, meeting it transversely in two points.

First, cut along the torus $T^{2} \times\{0\}$, as shown in Figure 4 (left). This divides the manifold into two pieces. By cutting along the torus, the disk bounded by the component $L$ has been cut into two. Slice up the middle of each half-disk and open it out, flattening it onto $T^{2} \times\{0\}$, as in Figure 4 (middle). Each half-disk has been sliced open into two parts, each of which is an $(r+1)$-gon. In Figure 4 (right), an example is shown for $r=5$. 

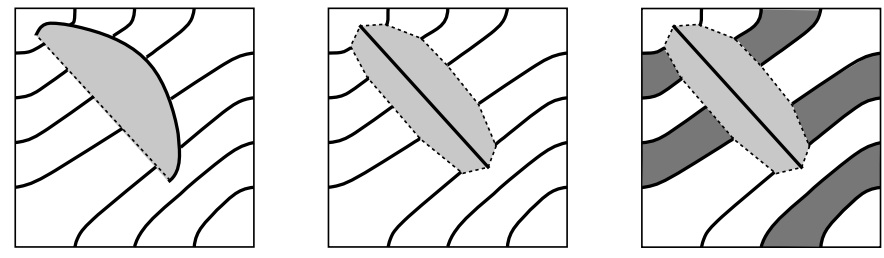

Figure 4 . To decompose $M(p, q, r, 0)$ into tetrahedra, a half-disk is sliced and flattened onto $T^{2} \times\{0\}$. Thick lines are components of the link. Dotted lines are edges of a polyhedral decomposition. One or two polygons remain after collapsing the shaded bigons.

Outside the flattened half-disk, regions are bigons, which we collapse to ideal edges as in Figure 4 (right), and either two quadrilaterals or a single hexagon adjacent to the ends of the half-disks. This can be seen as follows. View $T^{2} \times\{0\}$ as a rectangle with the usual side identifications to form the torus. Any region $U$ outside the halfdisk (as in Figure 4 (middle)) will either meet a single edge of the half-disk, two edges if $U$ is adjacent to an end of the half-disk, or zero edges if $U$ is not adjacent to the half-disk. Such regions are glued according to the identifications for a torus. Consider the regions meeting ends of the half-disk. Each of these two regions is glued to exactly two other regions. Either each of the two regions glue up to regions meeting only a single edge of the half-disk, in which case both regions are quadrilaterals, or the two regions may glue to meet each other. In this second case, the regions must additionally glue to regions meeting exactly one edge, so we have a hexagon.

The case of two quadrilaterals is the case of Figure 4. Since we obtain a hexagon only when both ends of the flattened half-disk belong to the same complementary region, this occurs if and only if $n+m=r$. Finally, any remaining regions meet one or zero edges, and must glue to form bigons; two bigons are shaded dark in Figure 4 (right).

Now cone each sliced half-disk, quadrilateral, and hexagon to the boundary component $T^{2} \times\{1\}$, as well as to the boundary component $T^{2} \times\{-1\}$. This gives a decomposition of the manifold $M(n, m, r, 0)$ into pyramids. Since the half disks are identified to each other and the outside regions are identified along the two pieces, the pyramids are glued in pairs along the regions on $T^{2} \times\{0\}$. By Lemma 3.3, we may subdivide into:

(1) $2(r+1)$ tetrahedra for two pairs of $(r+1)$-gons if $r>2$. If $r=2$, improve this to 4 tetrahedra.

(2) 6 tetrahedra for the single hexagon, if $n+m=r$. Otherwise, 8 tetrahedra for two quadrilaterals.

Observe that if $r=2$, then $n=m=1$ so we will have a hexagon in this case. Adding together these counts gives the result.

Lemma 3.5. Let $r>2$. Let $n, m, r$ and $s$ be integers such that $n<r, m<r$, $n+m \geq r$, and $0<s<r$. For any positive root $\beta \in B_{r}$, the manifold $M(n, m, r, s)$ 


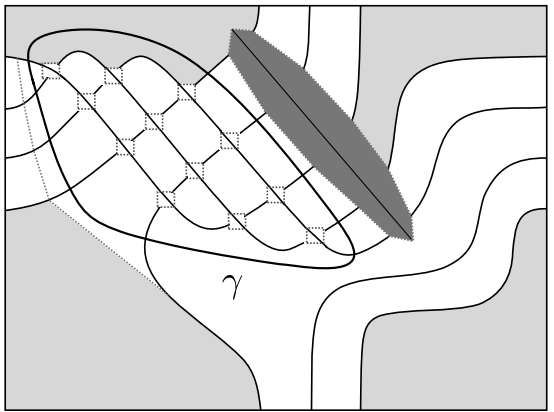

(a)

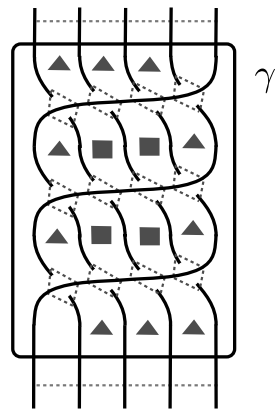

(b)

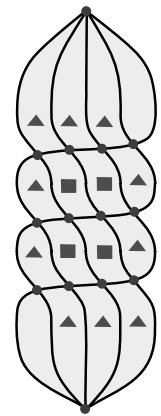

(c)

Figure 5. (a) Decompose $M(n, m, r, s)$ into tetrahedra, here $r=5$, $s=3$ and $\beta=\bar{\delta}_{5}$. Thick lines are link components, dotted lines are polygon edges. This figure differs from Figure 4 (middle) only in the encircled region. (b) For $\beta=\bar{\delta}_{5}$, schematic figure for $\beta^{3}$ with triangles and quadrilaterals indicated. (c) The disk triangulated with the projection of $\beta^{3}$.

can be decomposed into at most $t$ ideal tetrahedra, where

$$
t= \begin{cases}r s+3 r-s+9, & \text { if } \beta=\delta_{r} \text { or } \bar{\delta}_{r}, \\ r s+6 r-s+3, & \text { otherwise. }\end{cases}
$$

Proof. This proof is similar in spirit to that of Lemma 3.4: we subdivide $M(n, m, r, s)$ into two "polyhedral" pieces along a 2-complex roughly corresponding to the projection torus $T^{2} \times\{0\}$. Each of these pieces can be subdivided into tetrahedra by coning to $T^{2} \times\{ \pm 1\}$.

As above, the disk bounded by $L$ gets cut into two, each half-disk gets sliced up the middle and flattened out into two $(r+1)$-gons on $T^{2} \times\{0\}$. However, when $s \neq 0$, we now have a braid $\beta^{s}$, which can be seen as crossings on the torus $T^{2} \times\{0\}$. Encircle the braid $\beta^{s}$ in $T^{2} \times\{0\}$ with a simple closed curve $\gamma$, separating this braid from the rest of the diagram. (See Figure 5.) Note that the diagram outside this curve $\gamma$ agrees with the diagram of $M(n, m, r, 0)$. From here, the argument will proceed in four steps:

Step 1. Count and characterize the polygons inside $\gamma$. This is done in Lemma 3.6.

Step 2. At each quadrilateral bounded by the projection of $\beta^{s}$, insert a medial tetrahedron, as in Figure 7.

Step 3. Glue the faces of medial tetrahedra to certain adjacent triangles. This "collapsing" process, carried out in Lemma 3.7 (see Figures 6 and 7), reduces the number of faces visible from $T^{2} \times\{ \pm 1\}$.

Step 4. Cone all remaining faces to $T^{2} \times\{ \pm 1\}$, and count the resulting tetrahedra. This will complete the proof.

To begin the argument, we add an edge for every crossing of $\beta^{s}$. We also add edges, called peripheral edges, at the start and end of the braid, as in Figure 5(b). Every face bounded by these edges must be a quadrilateral, a triangle, or a bigon. No other polygons can occur because $\beta=\sigma_{i_{1}} \sigma_{i_{2}} \cdots \sigma_{i_{n-1}}$ is a word formed by a permutation of the $n-1$ generators $\sigma_{1}, \ldots, \sigma_{n-1}$; hence in the word $\beta^{s}$, no generator $\sigma_{j}$ appears 

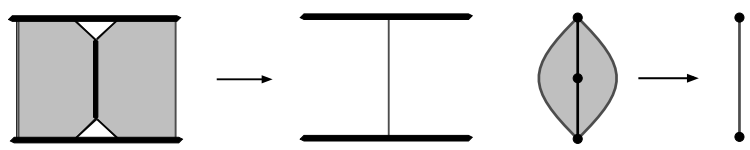

Figure 6. Collapsing two triangles with two common edges to an edge. Left: Shown on $T^{2} \times 0$ with edges of $\beta^{s}$. Right: Shown with ideal vertices.
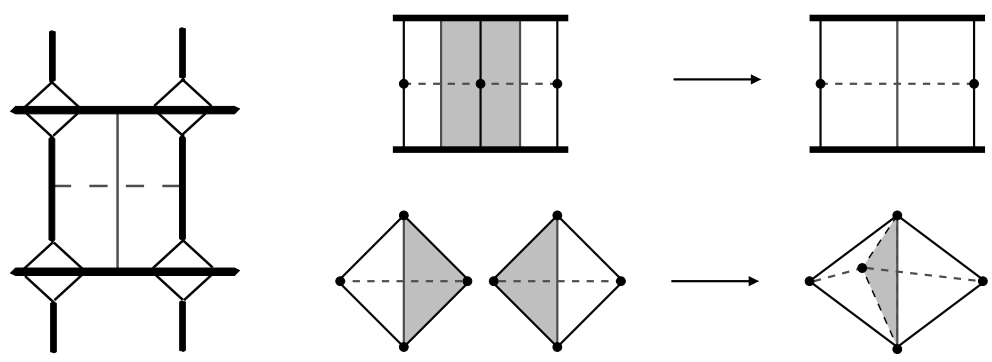

FIGURE 7. Left: medial tetrahedron inserted at every inner quadrilateral. Right: adjacent medial tetrahedra are glued together when their triangular faces with common edges are collapsed.

twice before a single appearance of $\sigma_{j+1}$ or $\sigma_{j-1}$. Moreover, bigons can only occur adjacent to the start or end of the braid.

Quadrilaterals and triangles that contain peripheral edges (i.e., that are adjacent to the start or end of the braid) will be called peripheral faces, and otherwise they will be called inner faces. Inner triangles can only occur on the sides of the region of $\beta^{s}$. Let $Q_{\mathrm{i}}, Q_{\mathrm{p}}, T_{\mathrm{i}}$ and $T_{\mathrm{p}}$ denote the number of inner quadrilaterals, peripheral quadrilaterals, inner triangles, and peripheral triangles, respectively. For example, in Figure 5(b), $Q_{\mathrm{i}}=4, Q_{\mathrm{p}}=0, T_{\mathrm{i}}=4, T_{\mathrm{p}}=6$.

Lemma 3.6. For any positive root $\beta \in B_{r}$, the region inside the curve $\gamma$ of $\beta^{s}$ contains

(1) $Q_{\mathrm{i}}=(r-3)(s-1)$ inner quadrilaterals,

(2) $Q_{\mathrm{p}} \leq(r-2)$ peripheral quadrilaterals, and

(3) $T_{\mathrm{p}}+Q_{\mathrm{p}} \leq 2(r-2)$ peripheral faces that are not bigons.

If $\beta=\delta_{r}$ or $\bar{\delta}_{r}$, there are no peripheral quadrilaterals and $T_{\mathrm{p}}=2(r-2)$ peripheral triangles.

Proof. Let $T=T_{\mathrm{i}}+T_{\mathrm{p}}$ and $Q=Q_{\mathrm{i}}+Q_{\mathrm{p}}$, and let $B$ be the number of bigons. The triangles, quadrilaterals, and bigons of $T^{2} \times\{0\}$ are nearly in one-to-one correspondence with triangles, quadrilaterals, and bigons of the projection graph of $\beta^{s}$, except at the start and end of the braid. We make the correspondence complete by pulling all strands at the start of the braid projection graph into a single vertex, and all strands at the end of the braid projection graph into a single vertex. The result is a graph on a 2-disk $D$, decomposed into quadrilaterals, triangles, and bigons. See Figure $5(\mathrm{c})$.

Let $c=s(r-1)$ denote the number of crossings of $\beta^{s}$. Let $v=2+c$ denote the number of vertices on $D$. Let $e=2 c+r$ be the number of edges, and $f=B+T+Q$ 
be the number of faces. Now, $\chi(D)=v-e+f=1$ implies $B+T+Q=c+r-1$. Moreover, $2 e=4 c+2 r=2 B+3 T+4 Q+2(s+1)$. We now subtract these equations:

$$
\begin{aligned}
& 2 B+3 T+4 Q=4 c+2 r-2 s-2 \\
& 3 B+3 T+3 Q=3 c+3 r-3 \\
& \hline-B+Q=c-r-2 s+1 .
\end{aligned}
$$

Using the formula $c=s(r-1)$, the last equation simplifies to

$$
Q=(B-2)+(r-3)(s-1) .
$$

We claim that $Q_{\mathrm{p}}=B-2$, which will be proved using the normal form for $\beta=$ $\sigma_{i_{1}} \sigma_{i_{2}} \ldots \sigma_{i_{n-1}}$, particularly Lemma 2.2 .

Note we obtain a bigon at the start of the braid $\beta^{s}$, between the $j$-th and $(j+1)$-st strands, $1<j<n-1$, if and only if in the word of $\beta, \sigma_{j}$ appears before both $\sigma_{j-1}$ and $\sigma_{j+1}$. For the $n$th and $(n+1)$-st strands, there will be a bigon if and only if $\sigma_{n-1}$ appears before $\sigma_{n-2}$. Finally, there will be a bigon between the first two strands if and only if $\sigma_{1}$ appears first in the word $\beta$. By Lemma 2.2, we pick up a bigon on top for the first chain in the indices of $\beta$, in equation (2.1), and one for each additional chain of length at least two.

Next, we obtain a quadrilateral at the start of $\beta^{s}$, between the $j$ th and $(j+1)$-st strands, $1<j<n-1$, if and only if $\sigma_{j}$ appears after both $\sigma_{j-1}$ and $\sigma_{j+1}$ in the word of $\beta$. There can be no quadrilaterals in the first or last strand positions. By Lemma 2.2, we pick up a quadrilateral for each chain in the indices of $\beta$ which has length at least two, except the first. In particular, the peripheral quadrilaterals at the start of the braid are in bijection with all but one of the bigons at the start of the braid. A similar analysis applies at the end of the braid.

Therefore, we conclude that the peripheral quadrilaterals are in bijection with all but two of the (peripheral) bigons, so that $Q_{\mathrm{p}}=B-2$. It follows from equation (3.1) that $Q_{\mathrm{i}}=(r-3)(s-1)$. Moreover, there are $r-1$ faces at the start of the braid, and $r-1$ faces at the end of the braid, so $Q_{\mathrm{p}}+T_{\mathrm{p}}+B=2(r-1)$. Thus,

$$
Q_{\mathrm{p}}+B \leq 2 r-2 \quad \Rightarrow \quad Q_{\mathrm{p}}+\left(Q_{\mathrm{p}}+2\right) \leq 2 r-2 \quad \Rightarrow \quad Q_{\mathrm{p}} \leq r-2 .
$$

Finally, at least two peripheral faces are bigons, so $Q_{\mathrm{p}}+T_{\mathrm{p}} \leq 2 r-4$. When $\beta=\delta_{r}$ or $\bar{\delta}_{r}$, there is only one chain in equation (2.1), hence $B=2, Q_{\mathrm{p}}=0$ and $T_{\mathrm{p}}=$ $2(r-2)$.

Recall that the root $\beta$ of $\Delta^{2}$ is a positive braid. Thus every inner quadrilateral, as in Lemma 3.6, is formed by two parallel over-strands crossing two parallel under-strands. At every inner quadrilateral, we insert a tetrahedron, called a medial tetrahedron. See Figure 7, (left). Two faces of every medial tetrahedron can be seen from $T^{2} \times\{1\}$ ("top"), and the remaining two faces can be seen from $T^{2} \times\{-1\}$ ("bottom").

We also insert a medial tetrahedron at every peripheral quadrilateral of the braid $\beta^{s}$. These look exactly the same as the tetrahedron in Figure 7, left, except with one corner truncated. Again, two faces can be seen from the top, and two from the bottom.

A consequence of inserting these medial tetrahedra is that when we view the region of $\beta^{s}$ from above (or below), all faces are triangles or bigons. Every bigon will collapse to an ideal edge, and almost all of the triangles can be glued in pairs: 
Lemma 3.7. Inside the region of $\beta^{s}$, let $t$ be a triangular face that is visible from the top and contains no peripheral edges, possibly a non-peripheral top face of a medial tetrahedron. Then $t$ shares two edges with an adjacent triangle $t^{\prime}$. The third edges of $t$ and $t^{\prime}$ are isotopic in $M(n, m, r, s)$. Thus $t$ can be glued to $t^{\prime}$, identifying their third edges. When this gluing operation is viewed from $T^{2} \times\{1\}$, the two triangles collapse to a single edge. See Figure 6.

A similar statement holds for a triangular face visible from $T^{2} \times\{-1\}$.

Proof. If $t$ is not peripheral, then all three sides of $t$ are inside the region of $\beta^{s}$ determined by $\gamma$. Because $\beta^{s}$ is a positive braid, two ideal edges of $t$ connect to a strand of the projection diagram between consecutive under-crossings. These two edges are shared with an adjacent triangle $t^{\prime}$, as in Figure 6.

Now, observe that every edge of $t$ is isotopic in $M(n, m, r, s)$ to a corresponding edge of $t^{\prime}$. Therefore, we may glue these two triangles together, by folding them toward the top. After the gluing, all that is visible from the top is a single ideal edge.

See Figure 7, (right), for an illustration of this gluing, in the case where $t$ and $t^{\prime}$ both belong to medial tetrahedra.

We now return to the proof of Lemma 3.5. As in the proof of Lemma 3.4, outside both the flattened half-disks and the curve $\gamma$ encircling $\beta^{s}$, regions on $T^{2} \times\{0\}$ are either bigons, in which case they collapse, or meet one or both of the intersections of the link $L$ with $T^{2} \times\{0\}$. In this case, the regions also meet the $s$ edges on either side of the $s$ overpasses. There are either two $(s+4)$-gons, or a single $(2 s+6)$-gon. One $(s+4)$-gon is shown as a shaded region in Figure $5(\mathrm{a})$.

Case: $\boldsymbol{\beta}=\boldsymbol{\delta}_{\boldsymbol{r}}$ or $\overline{\boldsymbol{\delta}}_{r}$. We now add $2(s-2)$ edges on $T^{2} \times\{0\}$, as shown in Figure 8 , subdividing the two $(s+4)$-gons or single $(2 s+6)$-gon into two hexagons or a single decagon, respectively, as well as $2(s-2)$ triangles adjacent to the braid. Viewed from the top, all inner faces can be glued in pairs to collapse to edges, and then triangles adjacent to the braid can also be glued in pairs, to collapse to a single edge. This is shown in the top row of Figure 8 . Thus, from the top, all that is left of the two $(s+4)$-gons or single $(2 s+6)$-gon are two hexagons or a single decagon, respectively, as in Figure 9. Viewed from the bottom, after the inner quadrilaterals are collapsed, the $2(s-2)$ new triangles remain, as well as the two hexagons or single decagon. When we cone to top and bottom, pyramids over the two hexagons or single decagon will be glued along those faces, and so we may perform stellar subdivision. Lemmas 3.3 and 3.6 apply, and we count:

(1) $2(r+1)$ tetrahedra from each pair of half-disks, which are $(r+1)$-gons.

(2) 12 tetrahedra from the two hexagons, or 10 tetrahedra from the single decagon.

(3) $(r-3)(s-1)$ medial tetrahedra.

(4) $2(r-2)$ tetrahedra from coning peripheral triangles to the top.

(5) $2(s-2)$ tetrahedra from coning other triangles to the bottom.

In this case, all the $(r-3)(s-1)$ medial tetrahedra are incident to the single collapsed edge seen from the top (the edge shown in the top right of Figure 8 and middle of Figure 9(a)).

Case: $\boldsymbol{\beta}$ is any positive root. For simplicity (and unlike the previous case), we will not attempt to collapse any additional triangles, beyond what was done in 

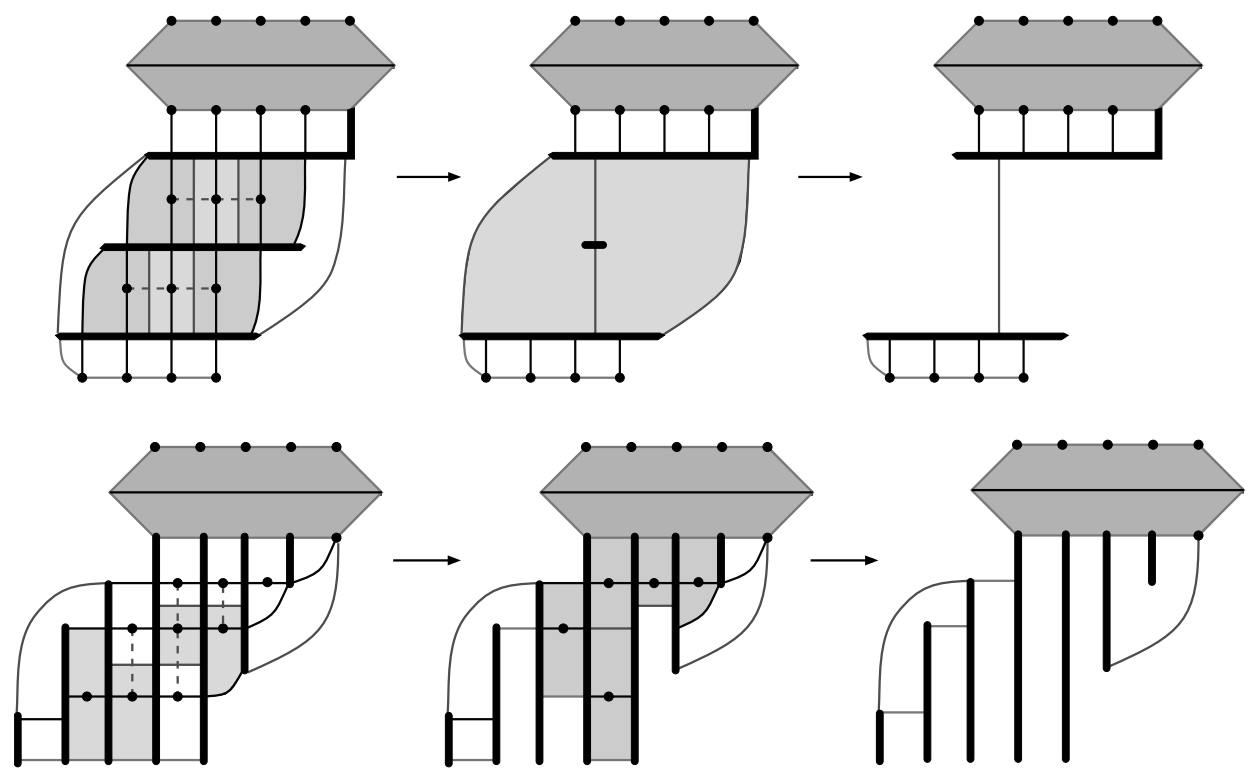

Figure 8. For a twisted torus braid with $s \neq 0$ and $\beta=\bar{\delta}_{r}$, attach medial tetrahedra as in Figure 6 right, and repeatedly collapse using Lemma 3.7. In the top row, $T^{2} \times\{0\}$ is seen from the top, and in the bottom row from the bottom. Thick black lines indicate ideal vertices.

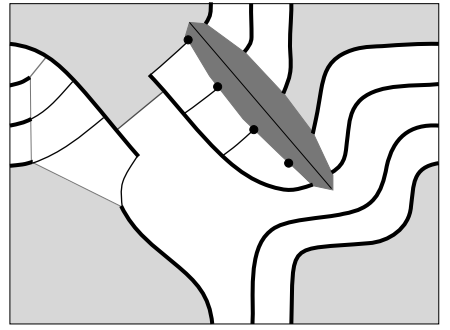

(a)

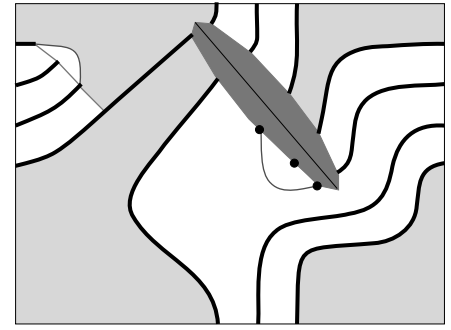

(b)

Figure 9. After collapsing as in Figure 8, regions on $T^{2} \times\{0\}$ seen from the top in (a) and from the bottom in (b).

Lemma 3.7. Instead, the two $(s+4)$-gons or the single $(2 s+6)$-gon will simply be coned to $T^{2} \times\{ \pm 1\}$, and subdivided into tetrahedra by stellar subdivision as in Lemma 3.3. Also, by Lemma 3.7, for each peripheral quadrilateral, only one triangular face of a medial tetrahedron will be visible from the top, and one from the bottom, after collapsing. Thus, peripheral triangles and quadrilaterals each contribute two tetrahedra by coning one triangle to the top, and one to the bottom. We count:

(1) $2(r+1)$ tetrahedra from each pair of half-disks, which are $(r+1)$-gons.

(2) $2(s+4)$ tetrahedra from stellar subdivision, assuming worst case of two $(s+4)$ gons.

(3) $Q_{\mathrm{i}}=(r-3)(s-1)$ medial tetrahedra from inner quadrilaterals. 
(4) $Q_{\mathrm{p}} \leq(r-2)$ medial tetrahedra from peripheral quadrilaterals.

(5) $2\left(Q_{\mathrm{p}}+T_{\mathrm{p}}\right) \leq 4(r-2)$ tetrahedra from coning peripheral faces to the top and bottom.

Adding these counts together completes the proof of Lemma 3.5.

We can now prove the upper volume bounds of Theorem 1.3.

Proof of Theorem 1.3. The link complement $S^{3} \backslash T(p, q, r, s)$ is obtained by Dehn filling the manifold $M(p, q, r, s)$, so $\operatorname{Vol}(T(p, q, r, s))$ is bounded above by the volume of $M(p, q, r, s)[16]$. By Lemma 3.2, the volume of $M(p, q, r, s)$ is the same as that of $M\left(m, n, r, s^{\prime}\right)$ or $M\left(n, m, r, s^{\prime}\right)$, where $s^{\prime}=s \bmod r, 0 \leq s^{\prime}<r$ and $n, m$ are as in the statement of that lemma.

The volume of any ideal tetrahedron is at most $v_{3}$. If $r=2$ and $s=0$, Lemma 3.4 implies that $M(1,1,2,0)$ can be decomposed into 10 ideal tetrahedra, hence $\operatorname{Vol}(M(1,1,2,0)) \leq 10 v_{3}$. Note that $M(1,1,2,1)$ differs from $M(1,1,2,0)$ by a single half-twist in a 2-punctured disk. Hence these two manifolds have the same volume [1]. Thus $\operatorname{Vol}(T(p, q, 2, s))<\operatorname{Vol}(M(1,1,2,0)) \leq 10 v_{3}$.

If $r>2$ and $s=0 \bmod r$, Lemma 3.4 implies the manifold $M(n, m, r, 0)$ (or $M(m, n, r, 0))$ can be decomposed into $2(r+4)$ tetrahedra, or $2(r+5)$ tetrahedra, depending on whether $n+m=r$ or not, respectively.

Finally if $r>2$ and $s \neq 0 \bmod r$, then Lemma 3.5 applies, and the manifold can be decomposed into at most $r s^{\prime}+3 r-s^{\prime}+9=(r-1) s^{\prime}+3 r+9$ tetrahedra if $\beta=\delta_{r}$, and $r s^{\prime}+6 r-s^{\prime}+3=(r-1) s^{\prime}+6 r+3$ otherwise. Since $0<s^{\prime}<r$, we obtain volume bounds $v_{3}\left(r^{2}+r+10\right)$ and $v_{3}\left(r^{2}+4 r+4\right)$, respectively.

When $r=2$, the bound of Theorem 1.3 is sharp. In the special case when $s=0$ $\bmod r$ and $m+n=r$, the above proof gives the better bound $v_{3}(2 r+8)$.

3.2. Links with volume approaching $10 v_{3}$. The following construction gives an explicit family of twisted torus knots with $r=2$ whose volumes approach $10 v_{3}$. This will prove Theorem 1.4, and demonstrate the sharpness of Theorem 1.3 when $r=2$.

Proof of Theorem 1.4. The manifold $S^{3} \backslash T(p, q, 2,2 N)$ is obtained by Dehn filling $M(p, q, 2,2 N)$. By Lemma $3.2, M(p, q, 2,2 N)$ is homeomorphic to $M(1,1,2,0)$. We begin the proof by showing that $M(1,1,2,0)$ is a hyperbolic manifold obtained by gluing 10 regular ideal tetrahedra, hence has volume exactly $10 v_{3}$.

Notice that the manifold $M(1,1,2,0)$ has a $\mathbb{Z}^{2}$ cover by the infinite chain-link-fence complement, Figure 10. The infinite chain-link-fence complement is studied in detail by Agol and Thurston in [13, Appendix]. In particular, they find a subdivision of this link complement into regular ideal tetrahedra.

This subdivision is obtained first by slicing the chain-link-fence complement in half along the projection plane, then slicing up half-disks bounded by crossing circles as in our proof of Lemma 3.4 above, and opening them up and flattening them. These are coned to points above or below the projection plane, yielding four tetrahedra per crossing disk. The remainder of the chain-link-fence complement consists of pyramids over regular hexagons. This is illustrated in Figure 17 of [13], where shaded triangles come from disks bounded by circles. To finish the decomposition into tetrahedra, Agol and Thurston replace two pyramids glued over a hexagon face with a stellar subdivision into six tetrahedra, as in Lemma 3.3. 


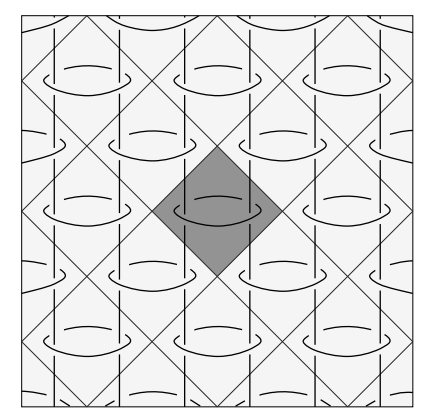

Figure 10. $M(1,1,2,0)$, shown in center, is covered by the infinite chain-link-fence complement.

All these tetrahedra in the decomposition of the chain-link-fence complement are now seen by a circle packing argument to be regular ideal tetrahedra. Notice that the subdivision is invariant under the action of $\mathbb{Z}^{2}$ corresponding to our covering transformation. Thus the regular ideal tetrahedra descend to give a decomposition of $M(1,1,2,0)$ into ideal tetrahedra. Tracing through the proof of Lemma 3.4, we see that these ideal tetrahedra agree with those of our decomposition. Since there are 10 such tetrahedra, the volume of $M(1,1,2,0)$ is $10 v_{3}$.

Another way to see that all tetrahedra are regular follows from the fact that all the edges of this triangulation are 6 -valent. In this case, the ideal tetrahedra satisfying the gluing equations have all dihedral angles $\pi / 3$, so they are regular ideal tetrahedra. Since all links of tetrahedra are equilateral triangles, they are all similar, and all edges of any triangle are scaled by the same factor under dilations. Hence, the holonomy for every loop in the cusp has to expand and contract by the same factor (i.e., it is scaled by unity), and so it is a Euclidean isometry. This implies that the regular ideal tetrahedra are also a solution to the completeness equations.

Finally, recall that every knot $T(p, q, 2,2 N)$ is obtained by Dehn filling three of the four boundary tori of $M(1,1,2,0)$. Two of these tori correspond to the components of the Hopf link, or equivalently the top and bottom boundary components of $T^{2} \times I$ in $M(1,1,2,0)$, while the third is the crossing circle encircling the two strands of the knot of slope $(1,1)$ on $T^{2} \times\{0\}$.

Now, choose a pair of (large) integers $p$ and $q$, such that $\operatorname{gcd}(p, q)=1$. In other words, there exist integers $(u, v)$, such that $u q-p v=1$. We may embed $T^{2} \times I$ into the complement of the Hopf link via the matrix

$$
A=\left[\begin{array}{ll}
u & p-u \\
v & q-v
\end{array}\right] . \text { Note that }\left[\begin{array}{ll}
u & p-u \\
v & q-v
\end{array}\right]\left[\begin{array}{l}
1 \\
1
\end{array}\right]=\left[\begin{array}{l}
p \\
q
\end{array}\right],
$$

hence this embedding sends the curve of slope $(1,1)$ on $T^{2}$ to a $(p, q)$ torus $\operatorname{knot}$ in $S^{3}$.

Consider the Dehn filling slopes in this construction. We will perform $(1, N)$ Dehn filling on the crossing circle in $T^{2} \times I$, thereby inserting $2 N$ crossings between a pair of strands in the $(p, q)$ torus knot. As for the top and bottom tori of $T^{2} \times I$, we will fill them along the slopes that become meridians of the Hopf link after embedding via the matrix $A$. In other words, in the original framing on $T^{2} \times I$, the top and bottom 


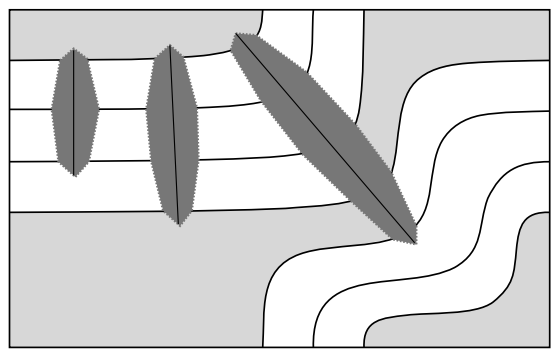

Figure 11. T-link with all $s_{i}^{\prime}=0$, and all half-disks flattened. $U$ is the shaded region. A polygon remains after collapsing bigons.

Dehn filling slopes are

$$
A^{-1}\left[\begin{array}{l}
1 \\
0
\end{array}\right]=\left[\begin{array}{c}
q-v \\
-v
\end{array}\right] \quad \text { and } \quad A^{-1}\left[\begin{array}{l}
0 \\
1
\end{array}\right]=\left[\begin{array}{c}
u-p \\
u
\end{array}\right] .
$$

Thus, so long as $N$ is large and $p=p_{N}$ and $q=q_{N}$ are also large, the Dehn filling slopes will be long. As $\left(p_{N}, q_{N}\right) \rightarrow(\infty, \infty)$, the length of the slopes approaches $\infty$. Thus the volume of the Dehn-filled manifold, $S^{3} \backslash T\left(p_{N}, q_{N}, 2,2 N\right)$, will approach $\operatorname{Vol}(M(1,1,2,0))=10 v_{3}$.

3.3. $\boldsymbol{T}$-links. Let $K$ be $T\left((p, q),\left(r_{1}, s_{1}\right), \ldots,\left(r_{k}, s_{k}\right)\right)$. Let $C_{1} \cup C_{2}$ be the Hopf link as above. For $i=1, \ldots, k$, augment the link $K$ with unknots $L_{i}$ that encircle the $r_{i}$ strands of the $i$ th braid $\beta_{i}$. Let $s_{i}^{\prime}=s_{i} \bmod r_{i}$, so that $0 \leq s_{i}^{\prime}<r_{i}$. Let

$$
M\left(p, q, r_{1}, s_{1}^{\prime}, \ldots, r_{k}, s_{k}^{\prime}\right)=S^{3} \backslash\left(C_{1} \cup C_{2} \cup\left(\cup_{i=1}^{k} L_{i}\right) \cup K\right) .
$$

Note $S^{3} \backslash K$ is homeomorphic to a Dehn filling on $M\left(p, q, r_{1}, s_{1}^{\prime}, \ldots, r_{k}, s_{k}^{\prime}\right)$.

Lemma 3.8. Suppose $s_{i}^{\prime}=0, i=1, \ldots, k$. Then $M\left(p, q, r_{1}, 0, \ldots, r_{k}, 0\right)$ can be decomposed into at most $r_{1}^{2}+9 r_{1}-8$ ideal tetrahedra.

Proof. As above, we will decompose $M\left(p, q, r_{1}, 0, \ldots, r_{k}, 0\right)$ into tetrahedra by first cutting the manifold into two pieces along the torus $T^{2} \times\{0\}$. The common boundary of these two pieces is shown in Figure 11. After cutting along $T^{2} \times\{0\}$, the punctured disks $D_{i}$ bounded by $L_{i}$ are cut into two, sliced and flattened onto $T^{2} \times\{0\}$. Each half-disk $D_{i}$ can be divided into two $\left(r_{i}+1\right)$-gons. We obtain $2\left(r_{i}+1\right)$ tetrahedra from each of these, as above, by first coning to the boundary $T^{2} \times\{1\}$ or $T^{2} \times\{-1\}$, obtaining two pyramids for each half-disk, and then applying Lemma 3.3. (If some $r_{i}=2$, we can improve this bound, but we won't use this fact.) This gives a total of $\sum_{i=1}^{k} 2\left(r_{i}+1\right)$ tetrahedra from half-disks.

Now we consider regions other than half-disks. Each such region is either a bigon, in which case it contributes no tetrahedra, or meets at least two edges on the end of some $D_{i}$. In particular, one region $U$ (the "top left corner" of Figure 11, shown shaded) will meet $2 k$ such edges, two for the end of each $D_{i}$ meeting in that region, as well as additional edges as the region connects to other regions by identifications on the torus.

The total number of tetrahedra for all these regions will be as large as possible when each region meets as few edges of the $D_{i}$ as possible. This will happen when 
$U$ meets only the $2 k$ edges corresponding to side edges of each $D_{i}$, and then just two more edges, either both from $D_{1}$ or one from $D_{1}$ and one from $D_{j}$, to close off. Additionally, the other end of each $D_{i}$ will be in a region meeting no other end of another disk and this region will meet exactly two edges from the end of $D_{i}$ and exactly two other edges of some other disks to close off. Hence each such region has four edges total. Thus when we have the maximum number of tetrahedra possible, we will have one region with $2 k+2$ edges, and $k$ quadrilaterals.

As before, cone these to pyramids lying above and below $T^{2} \times\{0\}$. Lemma 3.3 implies this can be divided into at most $(2 k+2)+4 k=6 k+2$ tetrahedra.

Since the $r_{i}$ 's are strictly decreasing and $r_{k} \geq 2$, it follows that $k \leq r_{1}-1$ and $\sum_{i=1}^{k} r_{i} \leq \sum_{i=2}^{r_{1}} i=\left(r_{1}^{2}+r_{1}-2\right) / 2$. Hence the number of tetrahedra is bounded by:

$$
\begin{aligned}
& \sum_{i=1}^{k} 2\left(r_{i}+1\right)+(6 k+2)=(6 k+2)+2 k+2 \sum_{i=1}^{k} r_{i} \\
& \quad \leq 8\left(r_{1}-1\right)+2+\left(r_{1}^{2}+r_{1}-2\right)=r_{1}^{2}+9 r_{1}-8 .
\end{aligned}
$$

This completes the proof of Lemma 3.8 .

Lemma 3.9. Suppose $s_{i}^{\prime} \neq 0$ for some $i, 1 \leq i \leq k$. Then $M\left(p, q,\left(r_{1}, s_{1}^{\prime}\right), \ldots,\left(r_{k}, s_{k}^{\prime}\right)\right)$ can be decomposed into $t$ ideal tetrahedra, where $t$ is at most $\frac{1}{3} r_{1}^{3}+\frac{5}{2} r_{1}^{2}+5 r_{1}-5$.

Proof. See Figure 12, which generalizes both Figures 5 and 11. First assume that $s_{i}^{\prime} \neq 0, \forall i=1, \ldots, k$. Each region with $\beta_{i}^{s_{i}^{\prime}}$, such as the $s_{i}^{\prime}$ overpasses in Figure 12, can be subdivided as in Lemma 3.6. Thus, we can repeatedly apply the methods of Lemma 3.5 in the general case that $\left\{\beta_{i}\right\}$ are any positive roots. We count:

(1) $\sum_{i=1}^{k} 2\left(r_{i}+1\right)$ tetrahedra from each pair of half-disks $D_{i}$.

(2) $\sum_{i=1}^{k}\left[\left(r_{i}-3\right)\left(s_{i}^{\prime}-1\right)+\left(r_{i}-2\right)\right]$ medial tetrahedra.

(3) $\sum_{i=1}^{k} 4\left(r_{i}-2\right)$ tetrahedra from coning remaining at most $2\left(r_{i}-2\right)$ peripheral triangles to the top and bottom.

To count the triangles in the unbounded regions in Figure 12, we recall from the proof of Lemma 3.8 that all regions outside the half-disks in Figure 11 together contribute at most $6 k+2$ tetrahedra. However, in Figure 12 , there are $2 \sum_{i=1}^{k} s_{i}^{\prime}$ additional side edges from the regions with overpasses. Because these side edges are adjacent,
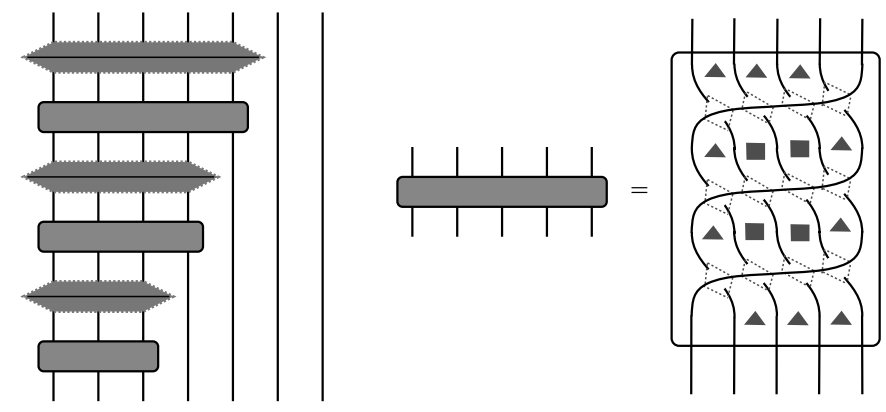

FiguRE 12. Schematic figure of a general $T$-link, with triangles and quadrilaterals indicated for the root $\bar{\delta}_{5}$. 
the number of unbounded regions in Figure 12 is the same as in the case where all $s_{i}^{\prime}=0$. Since we subdivide these regions into tetrahedra, one for each edge, it follows that the number of tetrahedra from these regions is at most the previous count plus the number of additional side edges: $6 k+2+2 \sum_{i=1}^{k} s_{i}^{\prime}$. (Whenever $\beta_{i}=\delta_{r_{i}}$ or $\bar{\delta}_{r_{i}}$, this triangulation can be improved by the methods of Lemma 3.5.)

Therefore, using $s_{i}^{\prime} \leq r_{i}-1$ and $k \leq r_{1}-1$, the total number of tetrahedra is bounded by:

$$
\begin{aligned}
t & \leq \sum_{i=1}^{k}\left(2\left(r_{i}+1\right)+\left(r_{i}-3\right)\left(s_{i}^{\prime}-1\right)+5\left(r_{i}-2\right)+2 s_{i}^{\prime}\right)+6 k+2 \\
& \leq \sum_{i=1}^{k}\left(2\left(r_{i}+1\right)+\left(r_{i}-3\right)\left(r_{i}-2\right)+5\left(r_{i}-2\right)+2\left(r_{i}-1\right)\right)+6 k+2 \\
& =\sum_{i=1}^{k}\left(r_{i}^{2}+4 r_{i}\right)+2 k+2 \leq \sum_{i=2}^{r_{1}}\left(i^{2}+4 i\right)+2 r_{1} \\
& =\frac{1}{3} r_{1}^{3}+\frac{5}{2} r_{1}^{2}+\frac{25}{6} r_{1}-5<\frac{1}{3} r_{1}^{3}+\frac{5}{2} r_{1}^{2}+5 r_{1}-5 .
\end{aligned}
$$

If $s_{i}^{\prime}=0$ for some $i, 1 \leq i \leq k$, this region does not contribute to the count of medial tetrahedra or tetrahedra from coning peripheral triangles. Hence, $t$ is bounded as above.

Proof of Theorem 1.7. Let $L$ be the following $T$-link

$$
L=T\left((p, q),\left(r_{1}, s_{1}, \beta_{1}\right), \ldots,\left(r_{k}, s_{k}, \beta_{k}\right)\right) .
$$

If $s_{i}=0 \bmod r_{i}$ for all $i$, then $S^{3} \backslash L$ is obtained by Dehn filling $M\left(p, q, r_{1}, 0, \ldots, r_{k}, 0\right)$, and by Lemma 3.8, $M$ can be decomposed into at most $r_{1}^{2}+9 r_{1}-8$ tetrahedra. Hence,

$$
\operatorname{Vol}(L)<v_{3}\left(r_{1}^{2}+9 r_{1}-8\right) \text {. }
$$

If some $s_{i} \neq 0 \bmod r_{i}$, then by Lemma 3.9, $S^{3} \backslash L$ is obtained by Dehn filling a manifold which decomposes into at most $\frac{1}{3} r_{1}^{3}+\frac{5}{2} r_{1}^{2}+5 r_{1}-5$ tetrahedra. Thus,

$$
\operatorname{Vol}(L)<v_{3}\left(\frac{1}{3} r_{1}^{3}+\frac{5}{2} r_{1}^{2}+5 r_{1}-5\right) .
$$

\section{Twisted torus knots with large volume}

In this section, we prove Theorem 1.8, showing that there exist twisted torus links with arbitrarily large volume.

Proof of Theorem 1.8. We will find a link $L_{N}$ in $S^{3}$ with volume at least $V+\varepsilon$, then show that twisted torus knots are obtained by arbitrarily high Dehn fillings of the components of the link. By work of Jørgensen and Thurston, for high enough Dehn filling we will obtain a twisted torus knot with volume at least $V$.

Consider again the Hopf link complement $S^{3} \backslash\left(C_{1} \cup C_{2}\right)$, which is homeomorphic to $T^{2} \times(-1,1)$. Consider $T^{2}$ as the unit square $[-1,1] \times[-1,1]$ with sides identified. Let $L$ be the link defined as the union of line segments $\{-1 / 2\} \times\{1 / 2\} \times[-1 / 2,1 / 2]$, $\{1 / 2\} \times\{-1 / 2\} \times[-1 / 2,1 / 2]$, and lines from $(-1 / 2,1 / 2)$ to $(1 / 2,-1 / 2)$ on the tori $T^{2} \times\{-1 / 2\}$ and $T^{2} \times\{1 / 2\}$. 


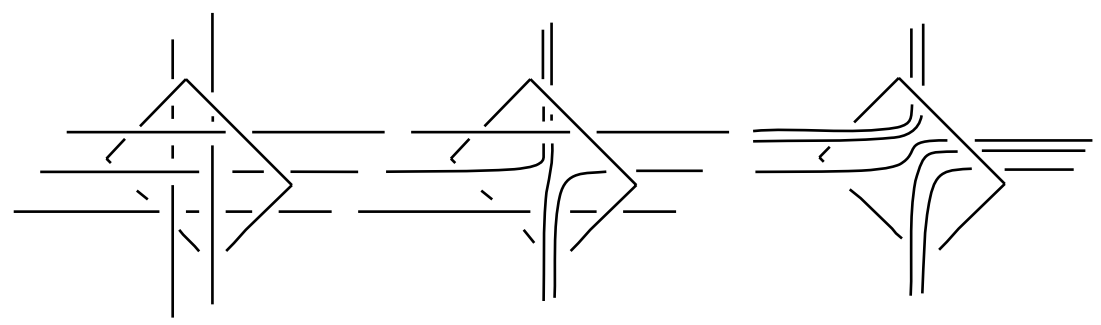

Figure 13. Left: portion of $L_{2}$ shown. Middle: Perform $1 / 2$ and $-1 / 2$ Dehn filling on $K_{1}$ and $K_{-1}$, respectively. Right: then perform 1 and -1 Dehn filling on $K_{2}$ and $K_{-2}$, respectively.

Chose $N \in \mathbb{Z}$ such that $v_{3}(2 N+4)>V$, where $v_{3}$ is the volume of a regular hyperbolic ideal tetrahedron. Let $K_{0}$ be the curve on $T^{2} \times\{0\}$ with slope 0 , running through the center $(0,0)$ of $T^{2}=[-1,1] \times[-1,1] / \sim$. Note $K_{0}$ does not meet $L$.

Now, for $i=1, \ldots, N$, if $i$ is odd, let $K_{i}$ and $K_{-i}$ be curves of slope 1/0 on $T^{2} \times\{i /(2 N+2)\}$ and $T^{2} \times\{-i /(2 N+2)\}$, respectively. If $i$ is even, let $K_{i}$ and $K_{-i}$ be curves of slope $0 / 1$ on $T^{2} \times\{i /(2 N+2)\}$ and $T^{2} \times\{-i /(2 N+2)\}$, respectively. Each $K_{ \pm i}$ is required to be a straight line on $T^{2}$, running through the center $(0,0)$ of $T^{2}=[-1,1] \times[-1,1] / \sim$. An example for $N=2$ is shown in Figure 13 (left).

Define the link $\widetilde{L}_{N}$ in $T^{2} \times(-1,1)$ by

$$
\widetilde{L}_{N}=\left(L \cup K_{0} \cup\left(\bigcup_{i=1}^{N}\left(K_{i} \cup K_{-i}\right)\right)\right) \text {. }
$$

We identify $S^{3} \backslash\left(C_{1} \cup C_{2}\right) \cong T^{2} \times(-1,1)$, and let $L_{N}$ denote $\widetilde{L}_{N} \cup C_{1} \cup C_{2}$ in $S^{3}$, so that $L_{N}$ is a link in $S^{3}$ with $4+2 N$ components.

Lemma 4.1. $S^{3} \backslash L_{N}$ is hyperbolic, for any $N$.

For readability, we will postpone the proof of Lemma 4.1 until we have finished proving Theorem 1.8. Assuming this lemma, since $S^{3} \backslash L_{N}$ is a hyperbolic manifold with $4+2 N$ cusps, its volume is at least $(4+2 N) v_{3}>V$ by work of Adams [2].

For any positive integers $n_{1}, \ldots, n_{N}$, perform Dehn filling on $L_{N}$ as follows. First, perform $1 / n_{1}$ Dehn filling on $K_{1}$ and $-1 / n_{1}$ filling on $K_{-1}$. The effect of this pair of Dehn fillings is to twist along the annulus bounded by $K_{1}$ and $K_{-1}$. See, for example, Baker [3] for an explanation of twisting along an annulus. Since $K_{0}$ is the only link component meeting this annulus, this Dehn filling performs $n_{1}$ Dehn twists of $K_{0}$ about the slope $1 / 0$ (corresponding to $K_{1}$ and $K_{-1}$ ), removes $K_{1}$ and $K_{-1}$, but otherwise leaves the link unchanged.

Now perform $1 / n_{2}$ filling on $K_{2}$, and $-1 / n_{2}$ filling on $K_{-2}$. Again the effect is a Dehn twist. Continue for each $i, i=1, \ldots, N$. The result is a manifold $M(p, q, r, 0)$, where $p / q$ has continued fraction expansion $\left[n_{1}, \ldots, n_{N}\right]$, and $r$ also depends on $N$ and the integers $n_{1}, \ldots, n_{N}$. See Figure 13. By choosing $n_{1}, \ldots, n_{N}$ to be large, we can ensure that $M(p, q, r, 0)$ is hyperbolic, and its volume is arbitrarily close to that of $S^{3} \backslash L_{N}$.

Now obtain a twisted torus knot by performing $1 / m$ Dehn filling on $L$ in $M(p, q, r, 0)$ and by performing Dehn filling on the Hopf link $C_{1} \cup C_{2}$ in $M(p, q, r, 0)$ along slopes 
with intersection number 1 . Since there are infinitely many of these, we may choose these slopes high enough that the result has volume arbitrarily close to that of $S^{3} \backslash L_{N}$. Since the volume of $S^{3} \backslash L_{N}$ is greater than $V$, this finishes the proof of Theorem 1.8.

Proof of Lemma 4.1. The proof is by induction. One can check (by drawing the link explicitly and triangulating by hand or computer [8]) that $S^{3} \backslash L_{1}$ is hyperbolic. The manifold $S^{3} \backslash L_{N}$ is obtained from $S^{3} \backslash L_{N-1}$ by removing the two closed curves $K_{N}$ and $K_{-N}$. We show that the manifold obtained by removing $K_{N}$ from $S^{3} \backslash L_{N-1}$ is hyperbolic, and similarly the manifold obtained by removing $K_{-N}$ from $S^{3} \backslash\left(L_{N-1} \cup\right.$ $\left.K_{N}\right)$ is hyperbolic, assuming hyperbolicity of the previous manifold. The proofs for $K_{N}$ and for $K_{-N}$ are identical, so we do them simultaneously. To establish notation, call the initial manifold $M_{N}$. Assuming $M_{N}$ is hyperbolic, we show that $M_{N} \backslash K$ is hyperbolic, where $K=K_{N}$ or $K=K_{-N}$. Recall that to show a link complement is hyperbolic, we need only show it is irreducible, boundary irreducible, atoroidal and an-annular.

First, note that $K$ cannot be homotopically trivial in $M_{N} \subset T^{2} \times I$, because it is parallel to the curve $1 / 0$ or $0 / 1$ on $T^{2}$.

Now it follows from standard arguments that $M_{N} \backslash K$ is irreducible and boundary irreducible, for any embedded essential 2-sphere in $M_{N} \backslash K$ would bound a ball in $M_{N}$, hence contain $K$, which would mean $K$ is homotopically trivial in $M_{N}$, contradicting the above paragraph. Any boundary compression disk would either have boundary on $K$, or would form half of an essential 2-sphere, in either case again implying $K$ is homotopically trivial. So $M_{N} \backslash K$ is irreducible and boundary irreducible.

If $M_{N} \backslash K$ contains an essential annulus, a regular neighborhood of the annulus and the link components on which its boundary lies gives an embedded torus in $M_{N} \backslash K$. If we can prove $M_{N} \backslash K$ is atoroidal, then again standard arguments will imply it is an-annular.

So it remains to show $M_{N} \backslash K$ is atoroidal. Suppose otherwise: there exists an essential torus $T$ in $M_{N} \backslash K$. Since $M_{N}$ is hyperbolic, $T$ is boundary-parallel or compressible in $M_{N}$. In either case, $T$ must bound a "trivial" three-dimensional submanifold $V \subset M_{N}$, where $V$ is one of the following:

(1) $V=T^{2} \times I$. This occurs when $T$ is boundary-parallel.

(2) $V$ is a solid torus.

(3) $V$ is a ball-with-knotted-hole, contained in a ball in $M_{N}$.

The last two cases occur if $T$ is compressible in $M_{N}$. In this case, surgering $T$ along its compressing disk $D$ will produce a sphere, which must bound a ball $B$ because $M_{N}$ is irreducible. If $B$ is disjoint from the compression disk $D$, then $V$ is obtained by adding a 1-handle whose cross-section is $D$, and is a solid torus. If $B$ contains $D$, then $V$ is obtained by removing the 1-handle whose cross-section is $D$, hence is a ball-with-knotted-hole.

It will help to consider the intersection between $T$ and the pair of pants $P$ whose three boundary components are a longitude of $K_{N-2}$, a meridian of $K_{N-1}$, and a longitude of $K_{N}=K$. ( $P$ forms the inner half of the 4 -punctured sphere depicted in Figure 14.) We assume that $P$ and $T$ have been moved by isotopy so as to minimize the number of curves of intersection. Because $P$ is incompressible, no curve of $P \cap T$ 


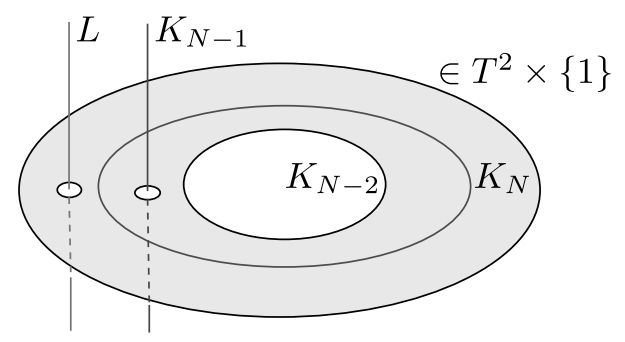

Figure 14. The knot $K=K_{N}$ lives on a four-punctured sphere in $M_{N}$.

can be trivial on $T$. Thus all curves of intersection run in parallel along some nontrivial slope in $T$. Note that the pair of pants $P$ contains only three isotopy classes of essential closed curve: these are parallel to the three boundary components. Because $P$ is essential, and the three boundary circles represent distinct elements of $\pi_{1}\left(M_{N}\right)$, the curves of $P \cap T$ that run in parallel on $T$ must also run in parallel along the same boundary component of $P$.

We consider the intersection $P \cap T$ to rule out the different types of trivial pieces enumerated above.

Claim 4.2. The torus $T$ cannot be boundary-parallel in $M_{N}$.

Proof of claim. Suppose that $T$ cuts off a product region $V=T^{2} \times I$, adjacent to a boundary component of $M_{N}$. Note that, since $T$ is essential in $M_{N} \backslash K$, we must have $K \subset V$. Consider whether $K_{N-2}$ and $K_{N-1}$ also intersect $V$.

If both $K_{N-2}$ and $K_{N-1}$ are disjoint from $V$, then $T$ separates $K$ from $K_{N-2}$ and $K_{N-1}$. But then the circles of $T \cap P$ run parallel to $K$, and $K$ is isotopic into $T$, hence into $\partial M_{N}$. This contradicts the construction of $K$.

If $K_{N-2}$ intersects $V$, then $V$ is parallel to the boundary component of $M_{N}$ that corresponds to $K_{N-2}$. On the other hand, $K_{N-1}$ must lie outside $V$, because all of $\partial V$ is already accounted for. Thus $T$ separates $K$ and $K_{N-2}$ from $K_{N-1}$, and the circles of $T \cap P$ run parallel to the meridian of $K_{N-1}$. But then there must be an essential annulus from the meridian of $K_{N-1}$ to the boundary torus corresponding to $K_{N-2}$. This contradicts the assumption that $M_{N}$ is hyperbolic.

If $K_{N-1}$ intersects $V$, then the argument is exactly the same, with $K_{N-1}$ and $K_{N-2}$ interchanged, and the longitude of $K_{N-2}$ in place of the meridian of $K_{N-1}$. Again, we get a contradiction.

Claim 4.3. The torus $T$ cannot bound a solid torus in $M_{N}$.

Proof of claim. Suppose that $T$ bounds a solid torus $V \subset M_{N}$. Then, because $T$ is incompressible in $M_{N} \backslash K$, we must have $K \subset V$. On the other hand, because all boundary components of $M_{N}$ are outside $V, K_{N-1}$ and $K_{N-2}$ must be outside $V$. Thus all circles of $T \cap P$ must be parallel to $K$. In particular, $K$ is parallel into the torus $T$.

Say that $K$ is an $(a, b)$ curve on $T$, which goes $a$ times around a meridian disk in $V$, and $b$ times around a longitude of $V$. Thus, in $\pi_{1}\left(T^{2} \times(-1,1)\right), K$ represents $b$ times the generator of $\pi_{1}(V)=\mathbb{Z}$. But by definition, $K=K_{N}$ is a $0 / 1$ or $1 / 0$ curve 
on $T^{2}$, which is primitive in $\pi_{1}\left(T^{2}\right)$. Therefore, $b= \pm 1$, and the $(a, \pm 1)$ curve $K$ is isotopic to the core of $V$.

We conclude that $T$ is the boundary of a regular neighborhood of $K$, contradicting the assumption that it is essential in $M_{N} \backslash K$.

Claim 4.4. The torus $T$ cannot be contained in a ball in $M_{N}$.

Proof of claim. Suppose that the trivial piece $V$ bounded by $T$ is a ball-with-knottedhole. Then $V$ is the complement of a tubular neighborhood of a knot $K^{\prime} \subset S^{3}$. Note that $K^{\prime}$ must truly be knotted, because by Claim 4.3, $V$ cannot be a solid torus.

Now, consider what happens to the pair $\left(M_{N}, K\right)$ when we Dehn fill all boundary components of $M_{N}$ along their meridians in $S^{3}$. Only the knot $K \subset S^{3}$ remains. By construction, $K$ is contained in $S^{3} \backslash V$, which is a tubular neighborhood of $K^{\prime}$. Furthermore, the torus $T$ is incompressible into $V$, and $K$ must intersect any compression disk of $T$ to the outside of $V$. Thus $T$ is incompressible in $S^{3} \backslash K$, and $K$ is a satellite knot with companion $K^{\prime}$.

On the other hand, recall that $K=K_{N}$ is a curve of slope $0 / 1$ or $1 / 0$ on the torus $T^{2}$, hence parallel to one of the components of the Hopf link, and an unknot in $S^{3}$. This is a contradiction.

By Claims 4.2, 4.3, and 4.4, T cannot be boundary-parallel or compressible in $M_{N}$. This violates the inductive hypothesis that $M_{N}$ is hyperbolic, and completes the proof that $M_{N} \backslash K$ is hyperbolic.

\section{Acknowledgments}

We thank the organizers of the 2007 conference at LSU, A second time around the Volume Conjecture, where this work was started. We thank the anonymous referee for the insightful idea that dramatically improved the tetrahedron count of Lemma 3.5. We thank Joan Birman for helpful comments. We are grateful for NSF support, and support for the first and third authors by PSC-CUNY.

\section{References}

[1] C. C. Adams, Thrice-punctured spheres in hyperbolic 3-manifolds, Trans. Amer. Math. Soc. $\mathbf{2 8 7}(2)$ (1985), 645-656.

[2] - Volumes of N-cusped hyperbolic 3-manifolds, J. London Math. Soc. (2) 38(3) (1988), $555-565$.

[3] K. Baker, Surgery descriptions and volumes of Berge knots. I. Large volume Berge knots, J. Knot Theory Ramifications 17(9) (2008), 1077-1097.

[4] J. Birman and I. Kofman, A new twist on Lorenz links, J. Topol. 2(2) (2009), 227-248.

[5] J. S. Birman, V. Gebhardt and J. González-Meneses, Conjugacy in Garside groups III: periodic braids, J. Algebr. 316(2) (2007), 746-776.

[6] P. J. Callahan, J. C. Dean and J. R. Weeks, The simplest hyperbolic knots, J. Knot Theory Ramifications 8(3) (1999), 279-297.

[7] A. Champanerkar, I. Kofman and E. Patterson, The next simplest hyperbolic knots, J. Knot Theory Ramifications 13(7) (2004), 965-987.

[8] M. Culler, N. M. Dunfield and J. R. Weeks, SnapPy, a computer program for studying the geometry and topology of 3-manifolds, http://snappy.computop.org.

[9] J. C. Dean, Small Seifert-fibered Dehn surgery on hyperbolic knots, Algebr. Geom. Topol. 3 (2003), 435-472.

[10] D. Futer, E. Kalfagianni and J. S. Purcell, Dehn filling, volume, and the Jones polynomial, J. Differ. Geom. 78(3) (2008), 429-464. 
1120 A. CHAMPANERKAR, D. FUTER, I. KOFMAN, W. NEUMANN AND J. S. PURCELL

[11] - Symmetric links and Conway sums: volume and Jones polynomial, Math. Res. Lett. 16(2) (2009), 233-253.

[12] - Cusp areas of Farey manifolds and applications to knot theory, Int. Math. Res. Not. 2010(23) (2010), 4434-4497.

[13] M. Lackenby, The volume of hyperbolic alternating link complements, Proc. London Math. Soc. (3) 88(1) (2004), 204-224. With an appendix by Ian Agol and Dylan Thurston.

[14] J. S. Purcell, Hyperbolic geometry of multiply twisted knots, Comm. Anal. Geom. 18(1) (2010), $101-120$.

[15] D. Rolfsen, Knots and links, Publish or Perish Inc., Berkeley, Calif. (1976), Mathematics Lecture. Series, No. 7.

[16] W. P. Thurston, The Geometry and Topology of Three-Manifolds, http://www.msri.org/ publications/books/gt3m/.

Department of Mathematics, College of Staten Island (CUNy), Staten Island, Ny 10314, USA

E-mail address: abhijit@math.csi.cuny.edu

Mathematics Department, Temple University, Philadelphia, PA 19122, USA

E-mail address: dfuter@temple.edu

Department of Mathematics, College of Staten Island (CUny), Staten Island, NY 10314, USA

E-mail address: ikofman@math.csi.cuny.edu

Department of Mathematics, Barnard College, Columbia University, New York, NY 10027, USA

E-mail address: neumann@math.columbia.edu

Mathematics Department, Brigham Young University, Provo, UT 84602, USA

E-mail address: jpurcell@math.byu.edu 